\title{
Integration ökologischer Parameter in das Reverse Network Design
}

\author{
Axel Tumaa, Lukas Meßmann*,a \\ *Address correspondence to: lukas.messmann@wiwi.uni-augsburg.de \\ ${ }^{a}$ Resource Lab, Institute of Materials Resource Management, University of Augsburg, \\ Universitaetsstr. 16, 86159 Augsburg, Germany
}

Veröffentlicht in: Dovbischuk I., Siestrup G., Tuma A. (Hrsg.): Nachhaltige Impulse für Produktion und Logistikmanagement, Springer Gabler, Wiesbaden, 189-203

https://doi.org/10.1007/978-3-658-21412-8 16

\begin{abstract}
Gegenstand der Arbeit ist die Analyse länderübergreifender Rücknahmenetzwerke für Elektroaltgeräte (EAG) unter Berücksichtigung ökonomischer und ökologischer Zieldimensionen. Hierzu wird das Netzwerkmodell eines europäischen IT-OEMs um ökologische Parameter erweitert, die auf der LCIAMethode ReCiPe basieren. Die Analyse umfasst aus der WEEE-Richtlinie und der Abfallverbringungsverordnung abgeleitete legislative Szenarien sowie unterschiedliche Aufkommensmengen. Die resultierenden Forschungsfragen werden anhand vier zentraler Erkenntnisse beantwortet, die sich auf die Aufkommensmenge, den Grad rechtlicher Einschränkungen sowie die Abwägung zwischen Transport- und Baukosten bzw. -umweltauswirkungen beziehen.
\end{abstract}




\section{Einleitung}

Die EU-Richtlinie zu WEEE (Waste Electric and Electronic Equipment) (EU 2012) verpflichtet Hersteller von IT-Geräten, diese nach der Nutzungsphase zurückzunehmen und aufzubereiten. Sie ist somit ein wichtiger Treiber für die Etablierung von Kreislaufwirtschaftssystemen. Neben gesetzlichen Verpflichtungen lassen sich auch relevante ökonomische und ökologische Gründe für die Rückführung von Produkten und Rohstoffen in die Wertschöpfungskette identifizieren. So stellt der Wiederverkaufswert gut erhaltener Elektro- und Elektronikaltgeräte (EAG) sowie der Anteil wertvoller Metalle ein entsprechendes wirtschaftliches Potential dar. Aus ökologischer Sicht reduziert eine Wiederverwendung den mit der Primärproduktion und der Produktion von elektronischen Bauteilen induzierten ökologischen Fußabdruck. Vor diesem Hintergrund werden Kreislaufwirtschaftssysteme (Closed-Loop Supply Chains) als nachhaltig per se wahrgenommen (Quariguasi Frota Neto et al. 2010).

Gleichzeitig gibt es einen großen Forschungsbedarf, ökologischen Nutzen und Grenzen von ClosedLoop Supply Chains zu bewerten. Stindt (2017) bietet einen umfassenden Überblick zu Literatur aus dem Bereich des nachhaltigen Supply Chain Managements, geht dabei besonders auf in der Literatur verwendete quantitative Methoden ein und präsentiert ein Rahmenwerk für nachhaltige Entscheidungsfindung. Brandenburg et al. (2014) sowie Ansari und Kant (2017) betonen den häufig qualitativen Charakter bisheriger Forschung und identifizieren einen Bedarf hinsichtlich quantitativer Methoden.

Die Minimierung ökologischer Auswirkungen wird insbesondere im Kontext des (Closed-Loop) Supply Chain Managements häufig mit der Minimierung des $\mathrm{CO}_{2}$-Ausstoßes gleichgesetzt. So verfolgen beispielsweise Bing et al. (2014), Zohal und Soleimani (2016), Chen et al. (2017) oder Nurjanni et al. (2017) das Ziel der Treibhausgasminimierung. Die europäische Umweltpolitik (EU 2008) zeigt eine weit größere Auswahl ökologischer Ziele auf. Neben der Vermeidung unnötigen Ressourcenverbrauchs stehen die Auswirkungen auf menschliche Gesundheit und Ökosysteme im Vordergrund, die wiederum von einer Vielzahl von Indikatoren, beispielsweise Versauerung, Landnutzung und -transformation oder Gewässereutrophierung, beeinflusst werden. Die bislang am weitesten entwickelte und anerkannteste Methode, um genannte Faktoren aggregiert zu betrachten, ist das Life Cycle Assessment (LCA) oder Ökobilanzierung (Europäische Kommission 2010). Azapagic and Clift (1999) wenden LCA im Rahmen einer Fallstudie im Bereich der Borproduktion an und integrieren die Ergebnisse als Koeffizienten in ein mehrkriterielles Optimierungsmodell, wobei auch hier der Fokus auf der Treibhausgasminimierung liegt. Dasselbe gilt für die Supply-Chain-Optimierung von Zhang et al. (2014). Hugo and Pistikopoulos (2005) dagegen integrieren LCA-basierte Umweltparameter in ein Standort- und Kapazitätsproblem. Eine nennenswerte Zahl von Studien verwendet LCAs, um Umweltaspekte im Bereich der Biokraftstoffproduktion (z.B. Santibañez-Aguilar et al. 2014, MurilloAlvardo et al. 2015, Cambero et al. 2016, Ren et al. 2016) oder anderer produktspezifischer vorwärtsgewandter Wertschöpfungsketten zu adressieren.

Vor diesem Hintergrund wird in der vorliegenden Arbeit ein Optimierungsmodell zur Gestaltung eines europäischen Rücknahme- und Aufbereitungsnetzwerks für Elektroaltgeräte erstellt und mit einem Solver gelöst. Dabei werden unterschiedliche legislative Szenarien und Aufbereitungsmengen betrachtet. Letztere hängen dabei im Wesentlichen von der Unternehmensgröße ab. Um dem 
europäischen Rahmen Rechnung zu tragen, wird dabei insbesondere auf die rechtlichen Implikationen der EU-Richtlinie zu WEEE (EU 2012) sowie der europäischen Abfallverbringungsverordnung (EU 2006) eingegangen. Die ökologische Bewertung erfolgt auf Basis von LCA-Daten.

Das Ziel dieser Arbeit ist daher die Beantwortung der folgenden Forschungsfragen:

* Welche Auswirkungen haben Aufkommensmenge und rechtliche Gegebenheiten auf die ökonomische und ökologische Bewertung von EAG-Aufbereitungsnetzwerken?

* Welche Konflikte oder Kongruenzen bestehen zwischen ökonomischer und ökologischer Zieldimension?

Die Arbeit untergliedert sich in die Beschreibung eines prototypischen Rücknahmenetzwerks für EAG (Reverse Network Design), sowie die Analyse und die Diskussion unterschiedlicher legislativer Szenarien und Aufkommensmengen.

\section{Reverse Network Design}

Zur Beantwortung der skizzierten Forschungsfragen wird das von Nuss, Stindt, Sahamie und Tuma (Nuss et al. 2016) entwickelte Reverse-Network-Design-Modell zur Analyse der Auswirkung verschiedener legislativer Szenarien im Rahmen einer Neugestaltung der europäischen Abfallverbringungsverordnung um ökologische Zieldimensionen erweitert. Das gemischt-ganzzahlige Optimierungsmodell basiert auf dem Praxisfall eines OEMs, der ein europäisches Rücknahmenetz für Elektroaltgeräte im B2B-Bereich evaluiert. Im Rahmen dieser Arbeit werden die ökonomischen Parameter um entsprechende ökologische, LCA-basierte ergänzt.

\subsection{Beschreibung des ökonomischen Modells}

Das ökonomische Basismodell ist aus der Perspektive eines europaweit agierenden OEMs formuliert, der gemäß der EU-Richtlinie zu WEEE (EU 2012) verpflichtet ist, Altgeräte zu sammeln und aufzubereiten. Hierzu werden Altgeräteaufkommen aus 26 Mitgliedstaaten der Europäischen Union (ohne Zypern und Bulgarien aufgrund fehlender Daten) betrachtet. Aus Komplexitätserwägungen werden die von Nuss et al. (2016) erhobenen Daten (NUTS-2) auf NUTS-1-Ebene (entspricht für Deutschland Bundesländern) aggregiert. Die prinzipiellen Modellvariablen betreffen folgende Entscheidungen:

- In welchen NUTS-1-Regionen führt der OEM die Sammlung von Altgeräten selbst durch, in welchen beauftragt er einen Logistikdienstleister?

- In welchen Regionen werden Sammelzentren (SZ) errichtet?

- In welchen Regionen werden Aufbereitungszentren (AZ) errichtet?

- Welche Technologie (einfache Trennung verwertbarer/nicht verwertbarer Fraktionen, Selektion nach Verwertungsoption [Recycling, Retrieval/Remanufacturing,]) wird in den regionalen SZ implementiert?

- Welche Technologie wird in den AZ implementiert (Demontage, Retrieval/Remanufacturing)?

- Welche Geräte werden direkt an den SZ an Drittverwerter verkauft, welche erst nach einer potentiellen Aufbereitung? 
- Welche Stoffströme entstehen als Resultat der genannten Entscheidungen zwischen Aufkommensregionen, Sammel- und Aufbereitungszentren?

Die Zielfunktion beschreibt das Ergebnis aus Sicht des OEMs. Die Nebenbedingungen berücksichtigen Netzwerkflussbedingungen sowie die legislativen Szenarien. Das ökonomische Basismodell ist im Appendix beschrieben.

\section{2 Ökologische Modellerweiterung (LCA)}

Zur ökologischen Bewertung des vorgestellten Reverse-Network-Modells werden analog zu den zentralen ökonomischen Parametern (Erlöse, fixe und variable Kosten) ökologische Parameter definiert (vgl. Tabelle 1). Die ökologischen Erlöse repräsentieren Umweltauswirkungen der durch das Rücknahmenetzwerk vermiedenen Primärproduktion, bewertet durch das Life Cycle Impact Assessment.

Die Modellierung der notwendigen Prozesse zur Bewertung der ökologischen Parameter wird in SimaPro 8.0.5 mit Anbindung an Ecolnvent v3 durchgeführt. Stellvertretend für Elektroaltgeräte wird ein Desktopcomputer modelliert. Die Ergebnisse des Life Cycle Impact Assessments (LCIA) werden mittels der Methode ReCiPe 1.12 auf einen Endwert (endpoint) aggregiert. Da im Falle der ökologischen Zielfunktion ein OEM-seitiges Recycling im Verhältnis zur Inanspruchnahme eines spezialisierten Dienstleisters nicht sinnvoll erscheint, werden zusätzliche Nebenbedingungen eingeführt, die entsprechende Technologien beim OEM ausschließen.

Tabelle 1: Ökologische Parameter und ihre ökonomischen Pendants

\begin{tabular}{|c|c|c|}
\hline 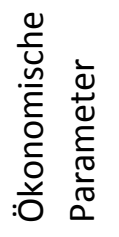 & 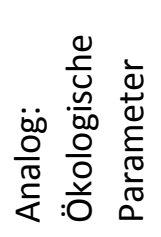 & Erläuterung \\
\hline$e r l^{S Z}$ & $\operatorname{prim}_{r}^{S Z}$ & $\begin{array}{l}\text { Umweltauswirkungen der durch den Verkauf an entsprechende } \\
\text { Dienstleister (sowie stoffliche Verwertung durch diese) an den SZ in } \\
\text { Region } r \text { vermiedenen Primärproduktion }\end{array}$ \\
\hline $\operatorname{erl}_{q}^{A Z}$ & $\operatorname{prim}_{q}^{A Z}$ & $\begin{array}{l}\text { Umweltauswirkungen der durch die Verwertung der } \\
\text { Sekundärprodukte/-rohstoffe an AZ vermiedenen } \\
\text { Primärproduktion, abhängig von Qualität } q\end{array}$ \\
\hline$f k_{a}^{S Z}$ & $f u_{a}^{S Z}$ & Umweltauswirkungen der Errichtung von SZ mit Technologie $a$ \\
\hline$f k_{b}^{A Z}$ & $f u_{b}^{A Z}$ & Umweltauswirkungen der Errichtung von AZ mit Technologie $b$ \\
\hline$v k^{N S}$ & $v u_{r}^{N S}$ & $\begin{array}{l}\text { Umweltauswirkungen von Abholungs- und Recyclingprozessen des } \\
\text { Dienstleisters (Nicht-Sammlung) in Region } r\end{array}$ \\
\hline$v k^{\text {ents }}$ & $v u_{r}^{\text {ents }}$ & Umweltauswirkungen einer Entsorgung in Region $r$ \\
\hline$v k_{a}^{S Z}$ & $v u_{r a}^{S Z}$ & $\begin{array}{l}\text { Umweltauswirkungen von Sortier- bzw. Inspektionsprozessen in SZ } \\
\text { der Region } r \text {, abhängig von Technologie } a\end{array}$ \\
\hline
\end{tabular}




\begin{tabular}{ccl}
\hline$v k_{q}^{A Z}$ & $v u_{r q}^{A Z}$ & $\begin{array}{l}\text { Umweltauswirkungen von Recycling- bzw. Aufbereitungsprozessen } \\
\text { in AZ der Region } r, \text { abhängig von Qualität } q\end{array}$ \\
\hline$v k^{\Pi}$ bzw. $v k^{\pi}$ & - & Keine ökologische Entsprechung \\
\hline$s k$ und $t k$ & $t u$ & Umweltauswirkungen des Transports \\
\hline
\end{tabular}

\subsection{Szenarien}

Analog zu Nuss et al. (2016) werden 20 Szenariokombinationen definiert, die sich aus vier Mengenund fünf legislativen Szenarien zusammensetzen: Die Mengenszenarien MS1, MS2, MS3 und MS4 repräsentieren hierbei $0,8 \%, 1,6 \%, 2,4 \%$ und 3,2\% des europäischen Aufkommens an EAG (Eurostat 2017) und tragen somit Herstellern unterschiedlicher Größe und damit auch unterschiedlich hohen Rücknahmeverpflichtungen Rechnung. Die legislativen Szenarien (LS) repräsentieren unterschiedliche Gesetzgebungen bzw. herstellerseitige Schlussfolgerungen in Bezug auf die Abfallverbringungsverordnung. Diese regelt die grenzüberschreitende Abfallverbringungen, an denen EU-Staaten beteiligt sind (EU 2006, Eurostat 2014). Die fünf legislativen Szenarien werden durch zusätzliche Nebenbedingungen im Modell umgesetzt (s. Appendix).

Im ersten Szenario (LS1) scheuen Hersteller den bürokratischen Aufwand sowie die rechtliche Unsicherheit, die mit einer grenzüberschreitenden Abfallverbringung einhergehen. Sammlung und Aufbereitung finden somit nur auf nationaler Ebene statt. Im LS2 besteht die Möglichkeit, in Sammelzentren gesammelte Mengen an EAG für den grenzüberschreitenden Transport zu notifizieren, was mit einmaligen Kosten pro notifiziertem Land einhergeht. Im LS3 besteht diese Möglichkeit ebenso, allerdings werden für die hochwertige Aufbereitung bestimmte Geräte nicht als Abfall klassifiziert und müssen somit nicht notifiziert werden. Im vierten Szenario (LS4) besteht zusätzlich die Möglichkeit, ganze Regionen für eine grenzüberschreitende Sammlung zu notifizieren, was mit einmaligen Kosten pro notifizierter Region einhergeht. Im letzten untersuchten Szenario (LS5) werden alle mit der Sammlung bzw. Verbringung von Altgeräten verbundenen Einschränkungen aufgehoben.

\section{Szenarienanalyse und Diskussion}

Das entwickelte gemischt-ganzzahlige Optimierungsmodell wird in IBM ILOG CPLEX Optimization Studio 12.6.1.0 implementiert und mit ökonomischer und ökologischer Parametrisierung jeweils für alle 20 Szenariokombinationen gelöst. Durchgeführt wird die Optimierung auf einem Intel(R) Xeon(R) E5-2690 (64 Bit) mit $8 \times 2,90 \mathrm{GHz}$ und $64 \mathrm{~GB}$ RAM, wobei die Laufzeit je nach Szenario und Parametrisierung zwischen 70 und 4.860 Sekunden beträgt. Ohne Aggregation auf NUTS-1-Regionen ist das Modell mit ökologischer Parametrisierung nicht mit praktikabler Rechenzeit lösbar. Die Szenarienanalyse und der Vergleich zwischen ökologischen und ökonomischen Ergebnissen ergeben folgende prinzipiellen Erkenntnisse:

- Je größer der OEM (d.h. je größer das Mengenszenario), desto profitabler das Rücknahmenetzwerk. Das gilt für alle legislativen Szenarien sowie für beide Zieldimensionen.

- In den restriktiveren Szenarien LS1, LS2 und LS3 werden im ökologischen Fall deutlich mehr SZ als im ökonomischen errichtet. Da in diesen Szenarien die Sammlung von EAG in einem Land auch 
mindestens ein SZ erfordert, geht eine Erweiterung des Netzwerks um weitere Länder in den meisten Fällen auch mit einer Erhöhung der Zahl der SZ einher (s. Abb. 1).

- In den weniger restriktiven Szenarien LS4 und LS5, in denen eine grenzüberschreitende Sammlung erlaubt ist, ist es jedoch umgekehrt. Hier ist die Zahl der SZ bei ökonomischer Optimierung größer als bei ökologischer (Abb. 1). Grund hierfür ist, dass die Transportkosten einen größeren Anteil an den Gesamtkosten haben als die Umweltauswirkungen des Transports an den Gesamtumweltauswirkungen. Dadurch können im ökologischen Fall bei erlaubter transnationaler Sammlung (LS4 und LS5) größere Transportwege in Kauf genommen werden, wodurch wiederum auf die Errichtung zusätzlicher SZ samt verbundener Umweltauswirkungen verzichtet werden kann. Das steht im Widerspruch zur verbreiteten Meinung, eine Minimierung der Transportwege sei gleichbedeutend mit einer Verringerung der Umweltauswirkungen.

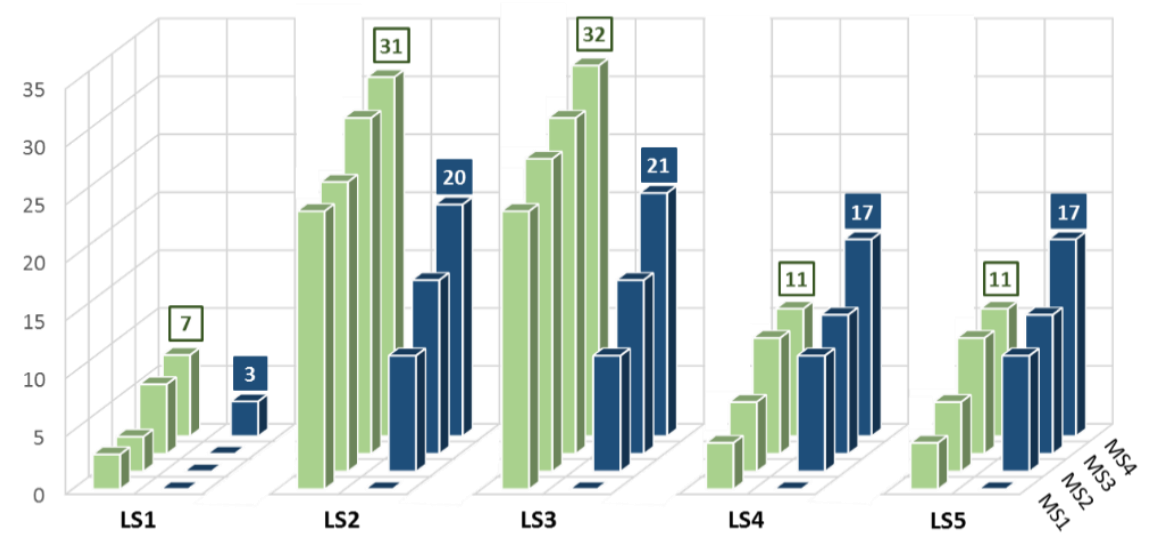

Abb. 1: Zahl der errichteten SZ für $\square$ ökol. und $\square$ ökon. Optimierung nach LS und MS

Die Auswertung der Zielfunktionswerte knüpft an die obigen Erkenntnisse an, nach der sich aus ökologischer Sicht die herstellerseitige Sammlung bereits bei kleineren Mengen lohnt. Abb. 2 zeigt, wie sich ökologische bzw. ökonomische Ergebnisse für beide Zieldimensionen verhalten. So führt beispielsweise in LS3 + MS3 das ökonomisch optimierte Netzwerk zu einem Gewinn von circa 4,5 Mio. $€(\mathbb{A})$ und zu ökologischen Einsparungen in Höhe von 21,1 Mio. Pt (B), während die ökologische Optimierung zu einem Ergebnis von -4,2 Mio. $€$ (C) und ökologischen Einsparungen von 21,9 Mio. Pt. (D) führt. Es wird deutlich, dass sich der Wert der ökologischen Einsparung in relaxierten legislativen Szenarien mit höheren Aufkommensmengen für ökologische und ökonomisch optimale Netzwerke annähert. D.h. je weniger restriktiv das Szenario und je größer die Aufkommensmenge, desto näher kommt die ökonomische Optimierung auch an das ökologisch optimale Ergebnis heran. Umgekehrt gilt das jedoch nicht. Das ökologisch optimale Netzwerk schneidet in den meisten Fällen wirtschaftlich sehr viel schlechter ab als ein auf Gewinnmaximierung ausgerichtetes. Insbesondere in den Szenarien LS2 bis LS5 werden geringe ökologische Verbesserungen zu hohen Kosten erkauft.

Dennoch können Aufbereitungsnetzwerke je nach Menge und Restriktion sowohl ökologisch als auch wirtschaftlich vorteilhaft sein. Die Frage ist für die meisten Szenariokombinationen also nicht ob, sondern wie ein solches Netzwerk gestaltet werden sollte. Entscheider müssen hierfür Zugriff auf adäquate Entscheidungshilfemodelle besitzen, um Trade-Offs identifizieren zu können. 


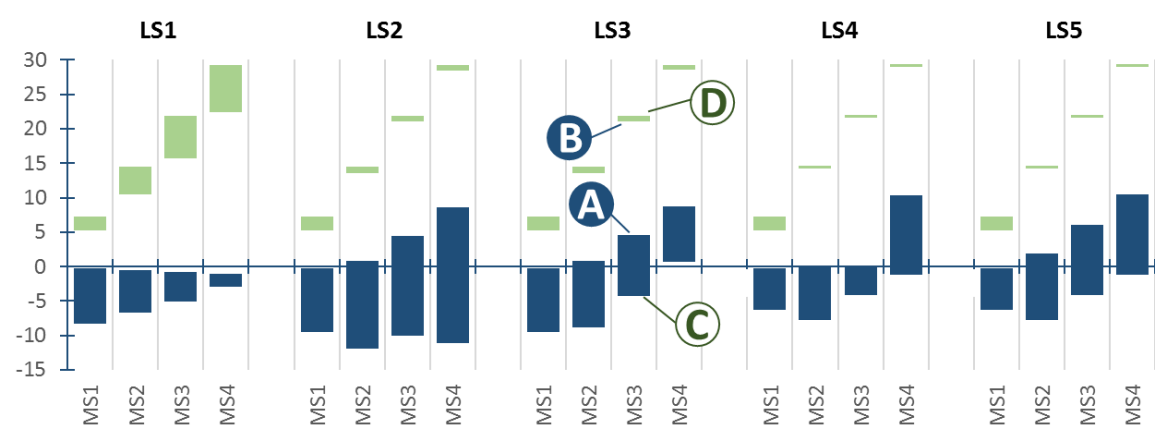

Abb. 2: Diskrepanz des @ökol. Ergebnisses (in Mio. Pt.) bzw. —ökon. Ergebnisses (in Mio. €) bei ökol. bzw. ökon. Optimierung

Diese Erkenntnisse spiegeln sich auch in der Menge wieder, die vom Hersteller direkt anstatt durch ein Drittunternehmen gesammelt wird. So entsteht bereits im ökologisch optimalen Fall in LS1 + MS1 jeweils ein SZ in Deutschland, Frankreich und dem Vereinigten Königreich, wodurch bereits mehr als $45 \%$ des gesamten Aufkommens gesammelt werden kann. Ab LS2 erhöht sich die Sammelquote bereits auf über $99 \%$, in LS4 wird das gesamte Aufkommen gesammelt. Im ökonomisch optimalen Fall hingegen lohnt sich eine herstellerseitige Sammlung in LS1 erst ab sehr hohen Sammelmengen (da eine vollständig an Drittunternehmen vergebene Sammlung zu noch höheren Kosten führen würde). Hier werden drei Sammelzentren in Deutschland errichtet, wodurch lediglich das deutsche Aufkommen gesammelt wird, was $18 \%$ des gesamten europäischen Aufkommens entspricht. Selbst in LS4 und LS5 werden Sammelquoten von über $90 \%$ erst ab MS2 erreicht. Abb. 3 und Abb. 4 zeigen die resultierende Netzwerkstruktur für zwei Szenarien und beide Zieldimensionen.

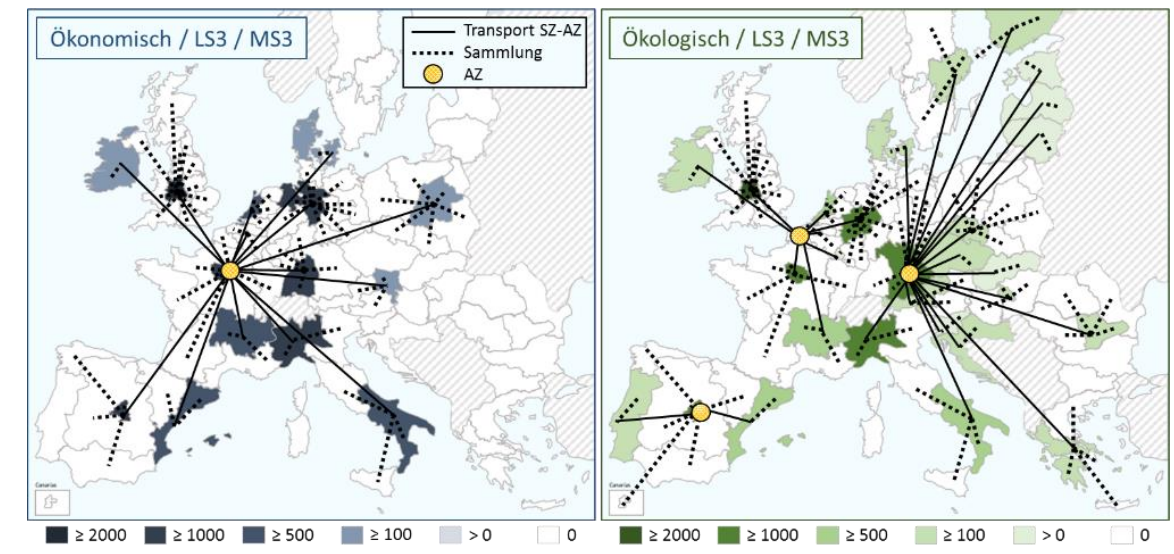

Abb. 3: Sammelmengen (in t), SZ-Standorte, AZ-Standorte \& Transportwege bei ökonomischer bzw. ökologischer Optimierung in LS3 + MS3 


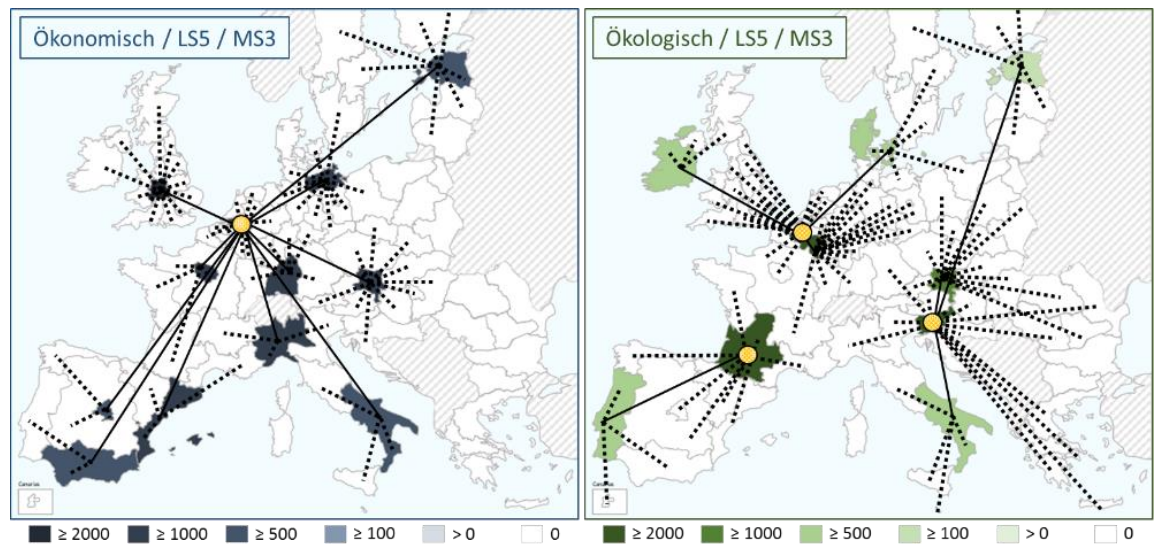

Abb. 4: Sammelmengen (in t), SZ-Standorte, AZ-Standorte \& Transportwege bei ökonomischer bzw. ökologischer Optimierung in LS5 + MS3

\section{Fazit}

Die vorliegende Arbeit untersucht die ökonomischen und ökologischen Implikationen eines europäischen Rücknahme- und Aufbereitungsnetzwerkes für EAG. Es werden optimale Standort- und Technologieentscheidungen aus der Sicht eines IT-OEMs analysiert. Dafür wird ein bestehendes gemischt-ganzzahliges Modell zur ökonomischen Optimierung um ökologische Parameter erweitert. Grundlage der Bewertung ist die LCIA-Methode ReCiPe 1.12. Das Optimierungsmodell wird für zwei Zieldimensionen (ökonomisch, ökologisch) in 20 Szenariokombinationen (fünf legislative und vier Mengenszenarien) gelöst. Ziel der Arbeit ist die Beantwortung der folgenden Forschungsfragen:

* Welche Auswirkungen haben Aufkommensmenge und rechtliche Gegebenheiten auf die ökonomische und ökologische Bewertung von EAG-Aufbereitungsnetzwerken?

* Welche Konflikte oder Kongruenzen bestehen zwischen ökonomischer und ökologischer Zieldimension?

In der Szenarienanalyse lassen sich vier zentrale Erkenntnisse identifizieren, anhand derer sich die Forschungsfragen beantworten lassen: 1) Höhere EAG-Aufkommensmengen führen sowohl aus ökologischer als auch aus ökonomischer Sicht zu bessern Zielfunktionswerten. 2) Aus ökologischer Sicht lohnt sich der Betrieb eines Netzwerkes gegenüber der Sammlung und Verwertung durch Drittanbieter bereits bei deutlich kleineren Mengen. 3) Im ökologischen Fall sind größere Transportwege dem Bau zusätzlicher Sammelzentren vorzuziehen. 4) Ökonomisch optimale Netzwerke besitzen in rechtlich wenig restriktiven Szenarien und bei großen Aufkommensmengen auch große ökologische Vorteile, während tatsächliche ökologische Optimalität mit wirtschaftlichen Verlusten einhergeht.

Insbesondere der letztgenannte Punkt zeigt den Bedarf nach weitergehender Forschung auf. Mehrkriterielle Optimierungsmodelle und -methoden (bspw. $\varepsilon$-Constraint, Goal Programming) müssen für den spezifischen Fall europäischer Aufbereitungsnetzwerke für EAG evaluiert und auf diesen angewendet werden. Sie können Entscheidungsträgern helfen, die Auswirkungen auf die Umwelt abzuschätzen sowie Zielkongruenzen und Trade-Offs zu identifizieren. 


\section{Literaturangaben}

Ansari ZN \& Kant R (2017) A state-of-art literature review reflecting 15 years of focus on sustainable supply chain management. Journal of Cleaner Production, 142(4):2524-2543

Azapagic A, Clift R (1999) Life cycle assessment and multiobjective optimization. Journal of Cleaner Production 7(2):135-143.

Bing X, Bloemhof-Ruwaard JM, van der Vorst, Jack GAJ (2014) Sustainable reverse logistics network design for household plastic waste. Flexible Service Manufacturing Journal 26(1-2):119-142

Brandenburg M, Govindan K, Sarkis J, Seuring S (2014) Quantitative models for sustainable supply chain management: developments and directions. European Journal of Operational Research 233(2):299-312

Cambero C, Sowlati T, Pavel M (2016) Economic and life cycle environmental optimization of forestbased biorefinery supply chains for bioenergy and biofuel production. Chemical Engineering Research and Design 107:218-235

Capitanescu F, Rege S, Marvuglia A, Benetto E, Ahmadi A, Navarrete Gutiérrez T, Tiruta-Barna L (2016) Cost versus life cycle assessment-based environmental impact optimization of drinking water production plants. Journal of Environmental Management 177:278-287

Chen Y, Wang L, Wang A, Chen T (2017) A particle swarm approach for optimizing a multi-stage closed loop supply chain for the solar cell industry. Robotics and Computer-Integrated Manufacturing 43:111-123

Europäische Kommission (2010) International Reference Life Cycle Data System (ILCD) Handbook General guide for Life Cycle Assessment - Detailed guidance. Publications Office, Luxembourg

Europäische Union (2006) Verordnung (EG) Nr. 1013/2006 des Europäischen Parlaments und des Rates vom 14. Juni 2006 über die Verbringung von Abfällen. ABI. L 190 vom 12.07.2006

Europäische Union (2008) Richtlinie 2008/98/EG des Europäischen Parlaments und des Rates vom 19. November 2008 über Abfälle und zur Aufhebung bestimmter Richtlinien., OJ L 312 vom 22.11.2008, 3-30

Europäische Union (2012) Richtlinie 2012/19/EU des Europäischen Parlaments und des Rates vom 4. Juli 2012 über Elektro- und Elektronik-Altgeräte. ABI. L 197 vom 24.07.2012, 38-71

Eurostat (2014) Grenzüberschreitende Abfallverbringung. http://ec.europa.eu/eurostat/de/web/waste/transboundary-waste-shipments. Zugriff: 15. Dezember 2017

Eurostat (2017) Waste statistics - electrical and electronic equipment. http://ec.europa.eu/eurostat/statistics-explained/index.php/Waste_statistics__electrical_and_electronic_equipment. Zugriff: 15. Dezember 2017

Gan VIL, Cheng JCP, Lo IMC (2016) Integrating life cycle assessment and multi-objective optimization for economical and environmentally sustainable supply of aggregate. Journal of Cleaner Production 113:76-85

Hugo A, Pistikopoulos EN (2005) Environmentally conscious long-range planning and design of supply chain networks. Journal of Cleaner Production 13(15):1471-1491

Murillo-Alvarado PE, Guillén-Gosálbez G, Ponce-Ortega JM, Castro-Montoya AJ, Serna-González M, Jiménez $L$ (2015) Multi-objective optimization of the supply chain of biofuels from residues of the tequila industry in Mexico. Journal of Cleaner Production 108(A):422-441

Nurjanni KP, Carvalho MS, Costa L (2017) Green supply chain design: A mathematical modeling approach based on a multi-objective optimization model. International Journal of Production Economics 183:421-432

Nuss C, Stindt D, Sahamie R, Tuma A (2016) Eine quantitative Analyse europäischer Richtlinien und Verordnungen zur Abfall- und Kreislaufwirtschaft am Beispiel der Elektro- und Elektronikindustrie: Implikationen und Empfehlungen für eine transnationale Umweltpolitik. Zeitschrift für Umweltpolitik \& Umweltrecht 39(1):37-69 
Park SY, Egilmez G, Kucukvar M (2016) Emergy and end-point impact assessment of agricultural and food production in the United States: A supply chain-linked Ecologically-based Life Cycle Assessment. Ecological Indicators 62:117-137

Quariguasi Frota Neto J, Walther G, Bloemhof J, van Nunen J, Spengler T (2009) A methodology for assessing eco-efficiency in logistics networks. European Journal of Operational Research 193(3):670-682

Quariguasi Frota Neto J, Walther G, Bloemhof J, van Nunen J, Spengler T (2010) From closed-loop to sustainable supply chains: the WEEE case. International Journal of Production Research 48(15):4463-4481

Ren J, An D, Liang H, Dong L, Gao Z, Geng Y, Zhu G, Song S, Zhoa W (2016) Life cycle energy and CO2 emission optimization for biofuel supply chain planning under uncertainties. Energy 103:151-166

Santibañez-Aguilara JE, González-Campos JB, Ponce-Ortega, JM, Serna-González M, El-Halwagi MM (2014) Optimal planning and site selection for distributed multiproduct biorefineries involving economic, environmental and social objectives. Journal of Cleaner Production 65:270-294

Stindt D (2017) A generic planning approach for sustainable supply chain management-How to integrate concepts and methods to address the issues of sustainability? Journal of Cleaner Production 153:146-163

Taskhiri MS, Garbs M, Geldermann J (2016) Sustainable logistics network for wood flow considering cascade utilization. Journal of Cleaner Production 110:25-39

Zhang Q, Shah, N, Wassick J, Helling R, van Egerschot, P (2014) Sustainable supply chain optimization: An industrial case study. Computers \& Industrial Engineering 74:68-83

Zohal M, Soleimani H (2016) Developing an ant colony approach for green closed-loop supply chain network design: a case study in gold industry. Journal of Cleaner Production 133:314-337

\section{Appendix}

Indexmengen:

$$
\begin{array}{ll}
L=\left\{1 \ldots N_{l}\right\} & \text { Länder, }|L|=26 \\
R=\left\{1 \ldots N_{r}\right\} & \text { Regionen, }|R|=92 \\
Q=\left\{1 \ldots N_{q}\right\} & \text { Qualitätsstufen des Aufkommensstroms, }|Q|=2 \\
A=\left\{1 \ldots N_{a}\right\} & \text { Technologien in Sammelzentren, }|A|=2 \\
B=\left\{1 \ldots N_{b}\right\} & \text { Technologien in Aufbereitungszentren, }|B|=2
\end{array}
$$

Allgemeine Daten:

$\begin{array}{ll}\text { allok }_{r l} & \text { 1, wenn Region } r(r \in R) \text { zu Land } l(l \in L) \text { gehört } \\ & 0 \text { sonst } \\ d_{r s} & \text { Distanz zwischen den Regionen } r \text { und } s(r, s \in R) \\ \text { auf }_{r} & \text { Aufkommen an EAG (in kg) in Region } r(r \in R) \\ \text { ant }^{\text {ents }} & \text { Anteil an Nicht-EAG, der im SZ entsorgt werden muss } \\ \text { ant }_{a q} & \text { Anteil an EAG, der im Sammelzentrum mit der Technologie } a(a \in A) \text { der } \\ & \text { Qualitätsstufe } q(q \in Q) \text { zugeordnet werden kann } \\ \text { BigM } & \text { Ausreichend große Zahl }\end{array}$


Ökonomische Parameter:

$\begin{array}{ll}s k & \text { Kosten für die Sammlung von EAG und den Transport zu einem SZ } \\ t k & \text { Kosten für den Transport von EAG von SZ zu AZ } \\ e r l_{q}^{S Z} & \text { Erlöse für aufbereitete EAG der Qualitätsstufe } q(q \in Q) \text { im SZ bei Verkauf an } \\ & \text { Drittunternehmen } \\ e r l_{q}^{A Z} & \text { Erlöse für aufbereitete EAG der Qualitätsstufe } q(q \in Q) \text { im AZ } \\ v k^{N S} & \text { Variable Kosten bei Nichtsammlung (Abholung und Verwertung durch } \\ & \text { Drittunternehmen) } \\ v k^{e n t s} & \text { Variable Kosten für die Entsorgung in SZ } \\ v k_{a}^{S Z} & \text { Variable Kosten für die Sortierung bzw. Inspektion von EAG in SZ } \\ v k_{q}^{A Z} & \text { Variable Kosten für Recycling bzw. hochwertige Aufbereitung von EAG mit } \\ f k^{\Pi} / f k^{\pi} & \text { Qualitätsstufe } q(q \in Q) \text { in AZ } \\ f k_{a}^{S Z} & \text { Kosten für die Notifizierung (Länder bzw. Regionen) } \\ f k_{b}^{A Z} & \text { Fixe Kosten für ein SZ mit Technologie } a(a \in A)\end{array}$

Entscheidungsvariablen:

$\begin{array}{ll}O_{r a}^{S Z} \in\{0,1\} & \text { Öffnung eines SZ in Region } r(r \in R) \text { mit Technologie } a(a \in A) \\ O_{r b}^{A Z} \in\{0,1\} & \text { Öffnung eines AZ in Region } r(r \in R) \text { mit Technologie } b(b \in B) \\ \Pi_{l m} / \pi_{r s} \in\{0,1\} & \text { Notifizierung von Land } l \text { nach Land } m(l, m \in L) \text { bzw. von Land } r \text { nach Land } \\ & S(r, s \in R) \text { (abhängig vom legislativen Szenario) } \\ W_{r s} \in\{0,1\} & \text { Sammlung des gesamten Aufkommens in Region } r \text { und Transport nach Region } \\ & S(r, s \in R) \\ \Delta_{r}^{W} \in\{0,1\} & \text { Sammlung und Verwertung des gesamten Aufkommens in Region } r(r \in R) \\ & \text { durch Drittunternehmen } \\ X_{r a} & \text { Menge an Altprodukten, welche in SZ in Region } r(r \in R) \text { mit SZ-Technologie } \\ & a(a \in A) \text { sortiert wird } \\ Z_{r s q} & \text { Menge an Altprodukten der Qualitätsstufe } q(q \in Q) \text {, die von SZ in Region } r \\ & \text { nach AZ in Region } s(r, s \in R) \text { transportiert wird } \\ \Delta_{r q}^{Z} & \text { Menge an Altprodukten der Qualitätsstufe } q(q \in Q) \text { aus SZ in Region } \\ & r(r \in R), \text { die von Drittunternehmen verwertet wird }\end{array}$

Zielfunktion:

maximiere

$=\sum_{r \in R} \sum_{s \in R} \sum_{q \in Q} Z_{r s q} \operatorname{erl}_{q}^{A Z}$

Erlöse in AZs

$+\sum_{r \in R} \sum_{q \in Q} \Delta_{r q}^{Z} e^{S Z}$

Erlöse in SZs

$-\sum_{r \in R} \sum_{s \in R} \sum_{q \in Q} Z_{r s q} v k_{q}^{A Z}$

Variable Kosten in AZs

$-\sum_{r \in R} \sum_{a \in A} X_{r a} v k_{a}^{S Z}$

Variable Kosten in SZs

$-\sum_{r \in R} \sum_{b \in B} O_{r b}^{A Z} f k_{b}^{A Z}$

Fixkosten für AZs

$-\sum_{r \in R} \sum_{a \in A} O_{r a}^{S Z} f k_{a}^{S Z}$

Fixkosten für SZs 
$-\sum_{r \in R} \Delta_{r}^{W}$ auf $k_{r} v k^{N S}$

$-\sum_{r \in R} \sum_{a \in A} X_{r a} a n t^{e n t s} v k^{\text {ents }}$

$-\sum_{r \in R} \sum_{s \in R} W_{r s} a u f k_{r} d_{r s} s k$

$-\sum_{r \in R} \sum_{s \in R} \sum_{q \in Q} Z_{r s q} d_{r s} t k$

$-\sum_{l \in L} \sum_{m \in L} \Pi_{l m} f k^{\Pi}$

$-\sum_{r \in R} \sum_{s \in S} \pi_{r S} f k^{\pi}$

Nebenbedingungen:

$\begin{array}{lll}\text { (I) } & \sum_{s \in R} W_{r s} \leq 1 & \forall r \in R \\ \text { (II) } & \Delta_{r}^{W}+\sum_{s \in R} W_{r s}=1 & \forall r \in R \\ \text { (III) } & W_{r r}=\sum_{a \in A} O_{r a}^{S Z} & \forall r \in R \\ \text { (IV) } & \sum_{a \in A} X_{s a}=\left(1-\Delta_{s}^{W}\right) a u f k_{s} & \forall s \in R \\ & +\sum_{r \in R} W_{r s} \text { aufk } & \\ & -\sum_{t \in R} W_{s t} \text { aufk } & \\ \text { (V) } & \Delta_{r q}^{Z}+\sum_{s \in R} Z_{r s q} & \forall r \in R, \forall q \in Q \\ & \sum_{a \in A} X_{r a} \text { ant }{ }_{a q} & \\ \text { (VI) } & \Delta_{r q}^{Z}+\sum_{s \in R} Z_{r s q} & \forall r \in R, \forall q \in Q \\ & \sum_{a \in A} O_{r a}^{S Z} \text { BigM } & \\ \text { (VII) } & W_{r s} \leq \sum_{a \in A} O_{s a}^{S Z} & \forall r, s \in R \\ \text { (VIII) } & W_{r s} \text { allo } k_{r l} \text { allok } k_{s m}=0 & \forall r, s \in R, \forall l, m \in L: l \neq m \\ \text { (IX) } & X_{r a} \leq O_{r a}^{S Z} B i g M & \forall r \in R, \forall a \in A \\ \text { (X) } & Z_{r s q} \leq \sum_{b \in B} O_{s b}^{A Z} B i g M & \forall r, s \in R, \forall q \in Q \\ \text { (XI) } & Z_{r s 2} \leq O_{r 2}^{A Z} B i g M & \forall r, s \in R \\ \text { (XII) } & \sum_{a \in A} O_{r a}^{S Z} \leq 1 & \forall r \in R \\ \text { (XIII) } & \sum_{b \in B} O_{r b}^{A Z} \leq 1 & \forall r \in R\end{array}$

Kosten für Fremdsammlung

Kosten für Entsorgung

Transportkosten für Sammlung

Transportkosten von SZ zu AZ

Notifizierungskosten für transnationalen Transport (nur legislative Szenarien 2, 3, 4)

Notifizierungskosten für transnationale Sammlung (nur legislatives Szenario 4)

Nebenbedingungen (legislative Szenarien):

$$
Z_{r s q} \text { allok }_{r l} \text { allo }_{s m}=0
$$$$
Z_{r s q} \text { allok }_{r l} \text { allok }_{s m} \leq \Pi_{l m} \text { BigM }
$$

$\forall r, s \in R, \forall q \in Q, \forall l, m \in L: l \neq m$

(LS3, LS4)

$$
Z_{r s 1} \text { allok }_{r l} \text { allok }_{s m} \leq \Pi_{l m} \text { BigM }
$$$$
\forall r, s \in R, \forall q \in Q, \forall l, m \in L: l \neq m
$$

$$
W_{r s} \text { allok }_{r l} \text { allok }_{s m} \leq \pi_{r s} \text { BigM }
$$

$\forall r, s \in R, \forall l, m \in L: l \neq m$

$\forall r, s \in R, \forall l, m \in L: l \neq m$ 